\title{
Neuropathology and Motor Behavior in Immature Rat Hypoxic-Ischemia
}

\author{
Elizabeth M. Jansen and Walter C. Low \\ Department of Neurosurgery and Graduate Program in Neuroscience, University of Minnesota, \\ Lions Research Building, 2001 6th Street SE, Minneapolis, MN 55455, USA
}

\section{INTRODUCTION}

Perinatal hypoxia and ischemia are major risk factors for motor disorders (e.g., cerebral palsy) in children. Currently there are no satisfying treatments for such disorders. We plan to assess the feasibility of neural transplantation to ameliorate the motor deficits characteristic of these conditions. In this study we employed a model of perinatal hypoxic-ischemia in the neonatal rat, originally developed by Levine /1/ and later modified by Rice $/ 2 /$, that replicates much of the neuropathology seen in cerebral palsy. The model consists of a permanent unilateral carotid artery ligation with a subsequent hypoxic exposure. This model creates unilateral damage in the striatum, hippocampus and overlying cortex ipsilateral to the ligation. To assess the efficacy of transplantation, a behavioral assay was established to measure the locomotor deficits induced by this model for pre- and post-transplant measurements. Behavioral performance was compared to severity of neuropathologic lesion. The potential for neural transplantation in this model is discussed.

\section{METHODS}

Unsexed seven-day old Sprague-Dawley rat pups were anesthetized with halothane and the right carotid artery was ligated with 6-0 surgical silk. Following surgery, the animals were returned to the dams for 1-3 hours, then placed in a hypoxic chamber (8\% oxygen, balance nitrogen) for three hours after which the animals were raised normally. Control animals underwent sham surgery with subsequent hypoxia or received a ligation alone. On postnatal day 30 , behavioral data were collected. We used a specialized instrument (Rota-Rod, Ugo Basile) which is a motorized treadmill designed to assess motor coordination in the rat. The length of time that the animals were able to stay on the treadmill on the first attempt was recorded and hypoxic-ischemic animals were compared to control animals. Behavioral performance was correlated with severity of neuropathologic lesion determined post mortem. The animals were then perfused transcardially with $4 \%$ paraformaldehyde and the brain were removed for glial fibrillary acidic protein (GFAP) staining (Histogen, Biogenex Laboratories).

\section{RESULTS}

The pathological lesions ranged from slight cytoarchitectural alterations to gross infarctions with dramatic loss of tissue. The less severe lesions exhibited cell loss and increased GFAP staining in the striatum (entire rostral-caudal extent), the hippocampal formation (CA2, CA3 $>\mathrm{CA} 1$ ) and the overlying motor cortices when compared qualitatively to the contralateral hemisphere or to controls. The more severe lesions showed gross unilateral infarctions in these areas leaving little or no overlying cortex. The mean latency for remaining on the treadmill (in seconds) was $100 \pm 51.7$ (S.E.M.) for animals with hypoxic-ischemic lesions $(n=8)$ and 202 \pm 43.4 for control animals $(n=7)(p<0.05)$. These results suggest that this locomotor task can serve as a functional assay for comparing locomotor coordination before and after neural transplantation in this model of neonatal rat hypoxicischemia. 


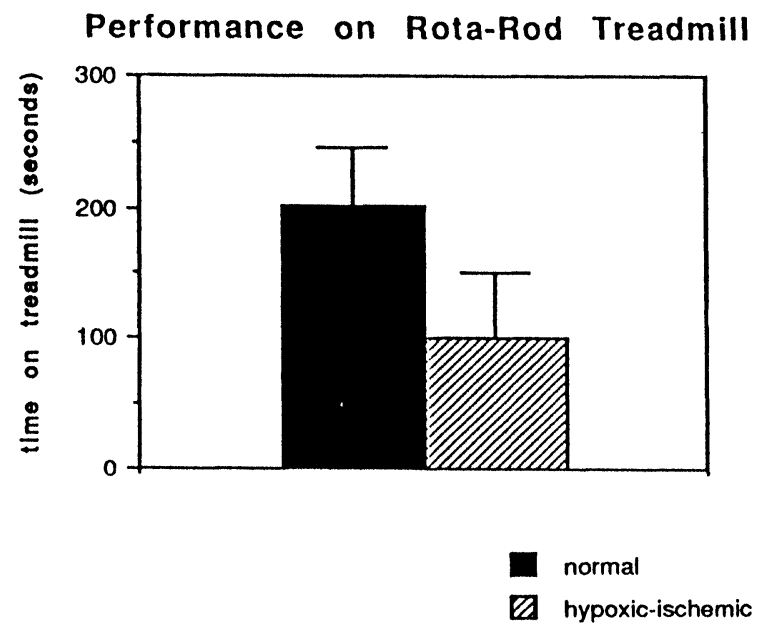

This work was supported in part by PHS Grant RO1-NS-24464 and a grant from the American Heart Association.

\section{REFERENCES}

1. Levine S. Am J Pathol 1960; 36: 1-17.

2. Rice JE, Vannucci RC, Brierly JB. Ann Neurol 1981; 9: 131-141.

ש hypoxic-ischemic 

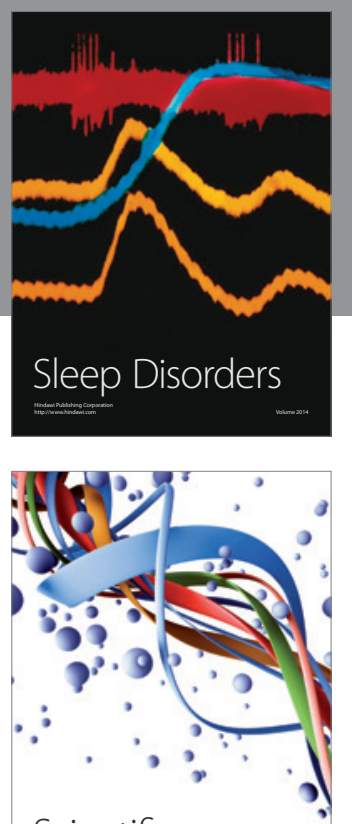

Scientifica
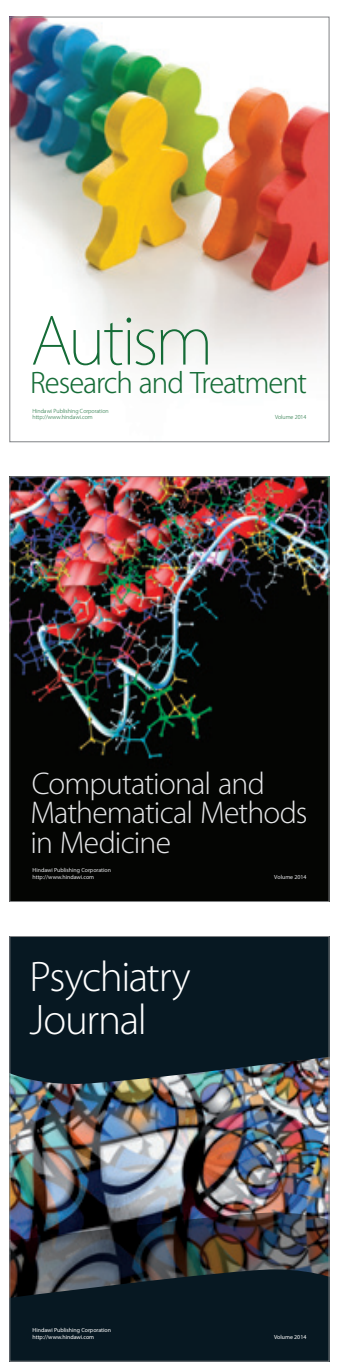
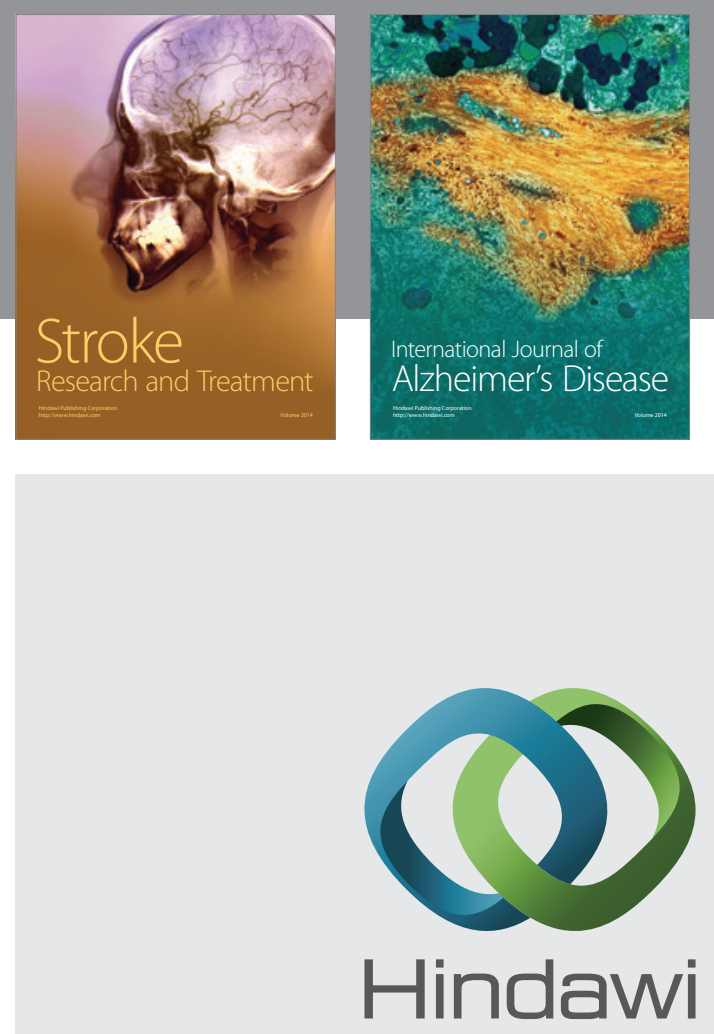

Submit your manuscripts at

http://www.hindawi.com
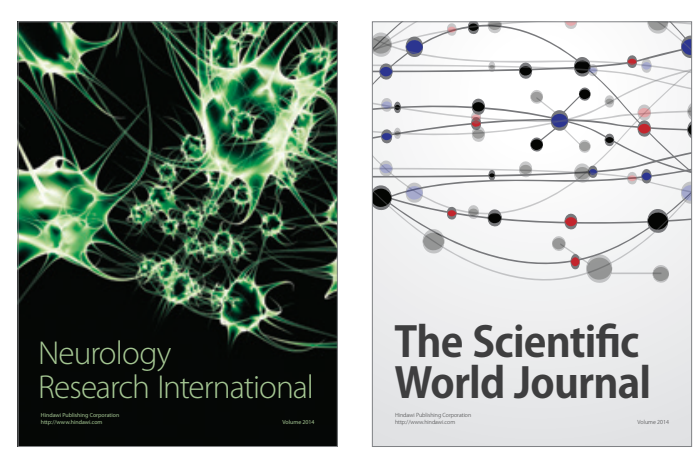

The Scientific World Journal

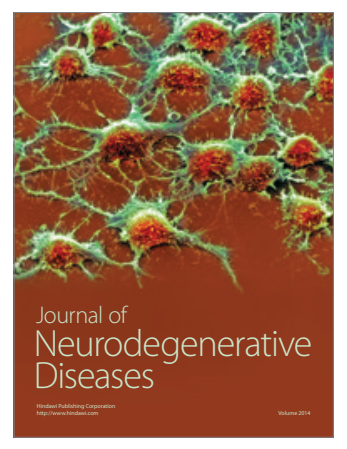

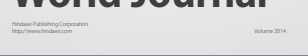

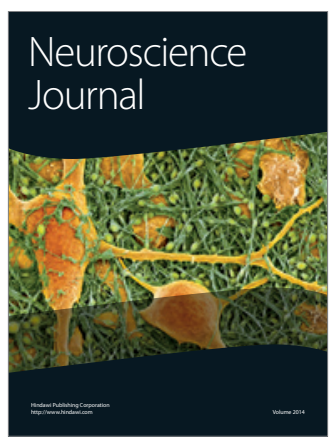

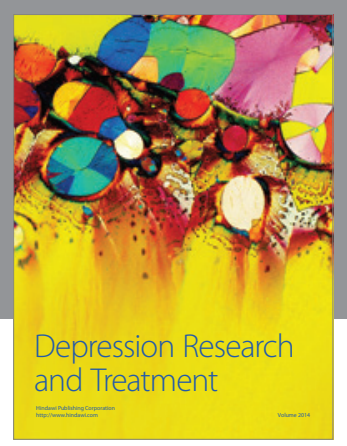
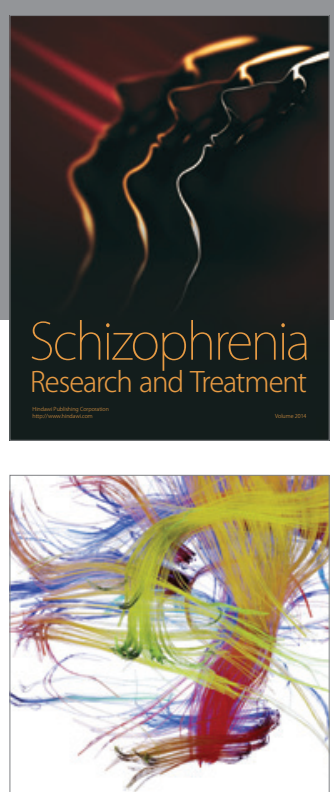

Brain Science

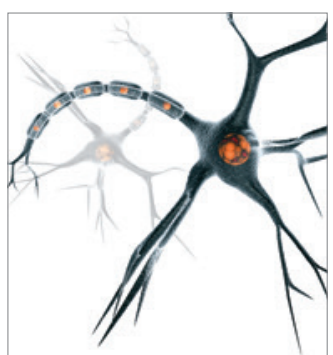

Neural Plasticity
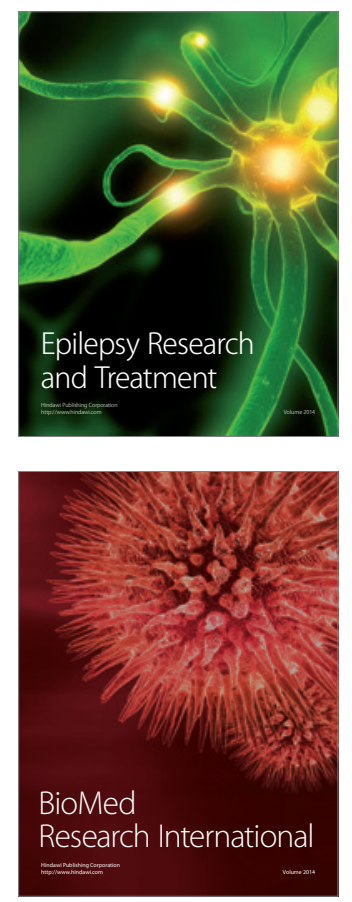

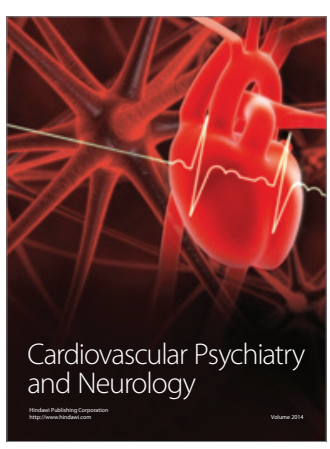

Parkinson's

Disease
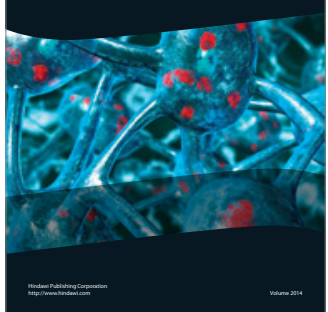